\title{
Obstetric pain: visual analog scale (VAS) and numerical rating scale (NRS) are correlated and have a modest but comparable sensitivity to detect a relief after epidural analgesia (EA)
}

Pratici E., Nebout S., Ghanem S., Malinas A., Hajage D., Keita H. Hôpital Louis Mourier-APHP-Paris 7 University, Dept of Anaesthesiology, Colombes, France

\section{Background:}

Excluding obstetric context, it has been shown that VAS and NRS were correlated, but this correlation (C) was stronger for lower pain scores compared with the highest scores (1).

It has also been described a difference between the two scales in their ability to detect pain relief after treatment (1). We conducted a survey on labor pain. The primary goal was

to evaluate the $C$ and the agreement between VAS and NRS and to evaluate the sensitivity of these two scales to detect a relief after EA. The $2^{\text {nd }}$ objective was to determine the preferred scale of patients.

\section{Materials/Methods:}

We conducted a prospective observational study in a category III maternity unit. Patients who wanted an EA for labor were included. $30 \mathrm{~min}$ before (BF) and after (AF) EA, patients were assessed by VAS and NRS at the peak of uterine contractions. $30 \mathrm{~min}$ $A F$ the EA, they indicated their relief level giving the\% pain reduction ( $R$ ). For VAS and NRS, the $\%$ of pain $R$ was calculated: $100 \mathrm{X}$ ((score BF EA - score AF EA) / score BF EA).

Demographic and obstetric data were collected. Statistical analysis by correlation coefficient of concordance (CCC) and graphics with Bland and Altman (BA) limits of agreement (1.96 $X S D)$.

Table: Parturients characteristics ( $n=97)$

\begin{tabular}{|c|c|}
\hline VARIABLE & Mediane $[\mathrm{QQR}], \mathrm{n}(\%)$ or Mean $\pm S D$ \\
\hline $\mathrm{AGE}(\mathrm{yr})$ & $31[29.35]$ \\
\hline$B M I\left(k g, m^{2}\right)$ & $24[21-26.6]$ \\
\hline Nulliparous & $67(69)$ \\
\hline Spontaneous labour/Induced labor & $75(77) / 22(23)$ \\
\hline Cervical dilatation at $\mathrm{E} /(\mathrm{cm})$ & $3[2-4]$ \\
\hline VAS before EA $(0-10)$ & $7.2 \pm 1.9$ \\
\hline NRS before EA $(0-10)$ & $7.6 \pm 1.7$ \\
\hline VAS 30 min after EA & $1.3 \pm 1.6$ \\
\hline NRS 30 min after EA & $1.6 \pm 1.7$ \\
\hline
\end{tabular}

BMI = Body mass index ; EA = Epidural analgesia; VAS = Visual Amalog Scale; NRS = Nunerical Rating Scale

\section{Results/Discussion:}

97 patients were included. Main results are inTable.The CCC $(95 \% \mathrm{Cl})$ between the\% $\mathrm{R}$ in pain reported by the patient and that calculated for VAS and NRS was respectively $0.7(0.6-0.8)$ and 0.73 (0.6-0.8). Analyses of BA BF and AF EA are represented by Fig1 and 2. The bias (agreement limits) between\% $R$ in the reported pain and that calculated for the VAS and NRS was respectively, 2.8 (31.2) and -1.2 (30.6). Preference was $16 \%$ and $66 \%$, respectively for VAS and NRS. $18 \%$ had no preference.

This study shows a strong $C$ between VAS and NRS for both severe pain and weak pain AF EA. The bias is small between the VAS and NRS either BFor AF EA since the average difference is close to 0 (-0.4 to -0.3). Two scales have a sensitivity to detect pain relief whith moderate $C$ with pain $R$ reported by patients.

\section{Figure 1, BA for VAS and NRS before $\mathrm{EA}$}

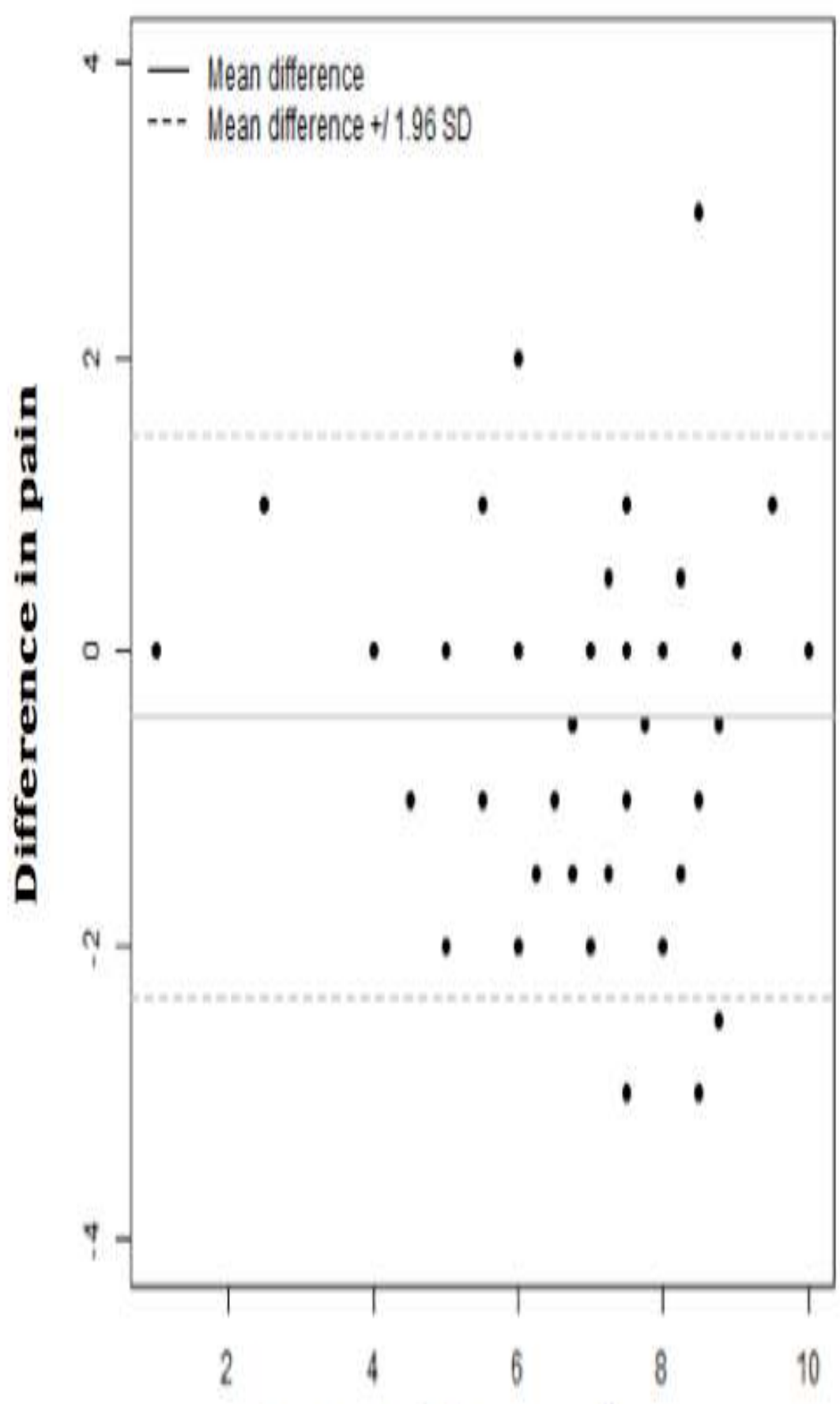

Average pain by two scales

\section{Conclusion:}

VAS and NRS are equivalent and either may be used to evaluate labor pain. NRS is parturients' preferred scale.

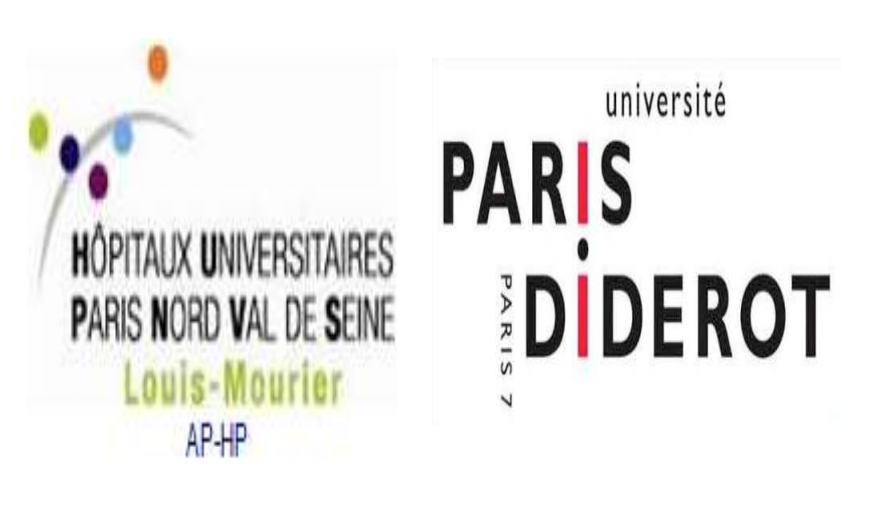

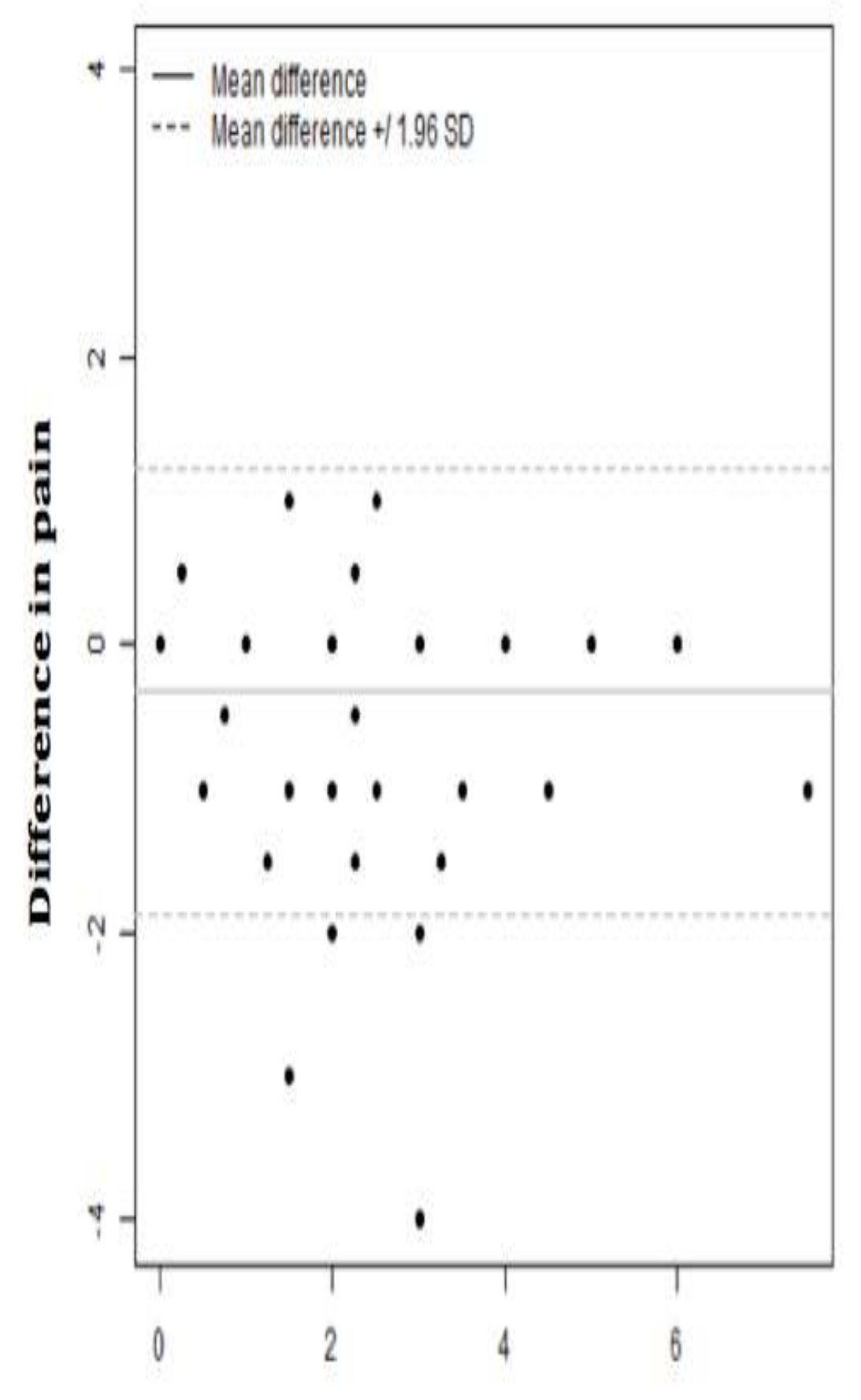

Average pain by two scales 